\title{
Article
}

\section{Existence of an integral operator and its consequences in fractional and conformable integrals}

\author{
Ghulam Farid ${ }^{1, *}$ \\ 1 Department of Mathematics, COMSATS University Islamabad, Attock Campus, Pakistan. \\ * Correspondence: ghlmfarid@ciit-attock.edu.pk
}

Received: 12 March 2019; Accepted: 11 July 2019; Published: 24 July 2019.

\begin{abstract}
The study of integral operators has always been important in the subjects of mathematics, physics, and in diverse areas of applied sciences. It has been challenging to discover and formulate new types of integral operators. The aim of this paper is to study and formulate an integral operator of a general nature. Under some suitable conditions the existence of a new integral operator is established. The boundedness of left and right sided integral operators is obtained and further boundedness of their sum is given. The investigated integral operators derive several known integrals and have interesting consequences for fractional calculus integral operators and conformable integrals. The presented results provide the boundedness of various fractional and conformable integral operators simultaneously.
\end{abstract}

Keywords: Integral operators, fractional integral operators, conformable integral operators, bounds.

MSC: 26D10, 31A10, 26A33.

\section{Introduction}

$\mathbf{T}$ he findings of this research are linked with integral operators in fractional calculus and conformable integrals, therefore we would like to give almost all related classical fractional integrals and conformable integral operators defined in recent decades. The well known Riemann-Liouville fractional integrals are defined as follows:

Definition 1. Let $f \in L_{1}[a, b]$. Then Riemann-Liouville fractional integral operators of order $\mu>0$ are defined as follows:

$$
{ }^{\mu} I_{a^{+}} f(x)=\frac{1}{\Gamma(\mu)} \int_{a}^{x}(x-t)^{\mu-1} f(t) d t, \quad x>a
$$

and

$$
{ }^{\mu} I_{b^{-}} f(x)=\frac{1}{\Gamma(\mu)} \int_{x}^{b}(t-x)^{\mu-1} f(t) d t, \quad x<b .
$$

$k$-fractional analogues of Riemann-Liouville fractional integral operators are given by Mubeen and Habibullah [1].

Definition 2. Let $f \in L_{1}[a, b]$. Then the $k$-fractional integrals of order $\mu, k>0$ are defined as follows:

$$
{ }^{\mu} I_{a^{+}}^{k} f(x)=\frac{1}{k \Gamma_{k}(\mu)} \int_{a}^{x}(x-t)^{\frac{\mu}{k}-1} f(t) d t, \quad x>a
$$

and

$$
{ }^{\mu} I_{b^{-}}^{k} f(x)=\frac{1}{k \Gamma_{k}(\mu)} \int_{x}^{b}(t-x)^{\frac{\mu}{k}-1} f(t) d t, \quad x<b .
$$

A more general definition of the Riemann-Liouville fractional integral operators is given as follows [2]: 
Definition 3. Let $f:[a, b] \rightarrow \mathbb{R}$ be a integrable function. Also let $g$ be an increasing and positive function on $(a, b]$, having a continuous derivative $g^{\prime}$ on $(a, b)$. The left-sided and right-sided fractional integrals of a function $f$ with respect to another function $g$ on $[a, b]$ of order $\mu>0$ are defined as:

$$
{ }_{g}^{\mu} I_{a^{+}} f(x)=\frac{1}{\Gamma(\mu)} \int_{a}^{x}(g(x)-g(t))^{\mu-1} g^{\prime}(t) f(t) d t, x>a
$$

and

$$
{ }_{g}^{\mu} I_{b^{-}} f(x)=\frac{1}{\Gamma(\mu)} \int_{x}^{b}(g(t)-g(x))^{\mu-1} g^{\prime}(t) f(t) d t, x<b,
$$

where $\Gamma($.$) is the Gamma function.$

A $k$-fractional analogue of above definition is given by Kwun et al. In 2018 [3].

Definition 4. Let $f:[a, b] \rightarrow \mathbb{R}$ be a integrable function. Also let $g$ be an increasing and positive function on $(a, b]$, having a continuous derivative $g^{\prime}$ on $(a, b)$. The left-sided and right-sided fractional integrals of a function $f$ with respect to another function $g$ on $[a, b]$ of order $\mu, k>0$ are defined as:

$$
{ }_{g}^{\mu} I_{a^{+}}^{k} f(x)=\frac{1}{k \Gamma_{k}(\mu)} \int_{a}^{x}(g(x)-g(t))^{\frac{\mu}{k}-1} g^{\prime}(t) f(t) d t, x>a
$$

and

$$
{ }_{g}^{\mu} I_{b^{-}}^{k} f(x)=\frac{1}{k \Gamma_{k}(\mu)} \int_{x}^{b}(g(t)-g(x))^{\frac{\mu}{k}-1} g^{\prime}(t) f(t) d t, x<b,
$$

where $\Gamma_{k}($.$) is the k$-gamma function.

A detail study of fractional and conformable integral operators which are investigated in recent years is summarized in the following remark:

Remark 1. Fractional integrals elaborated in (7) and (8) particularly produce several known fractional integrals corresponding to different settings of $k$ and $g$.

(i) For $k=1$ (7) and (8) fractional integrals coincide with (5) and (6).

(ii) For taking $g$ as identity function (7) and (8) fractional integrals coincide with (3) and (4).

(iii) For $k=1$, along with $g$ as identity function (7) and (8) fractional integrals coincide with (1) and (2).

(iv) For $k=1$ and $g(x)=\frac{x^{\rho}}{\rho}, \rho>0$, (7) and (8) produce Katugampola fractional integrals defined by Chen and Katugampola in 2017 [4].

(v) For $k=1$ and $g(x)=\frac{x^{\tau+s}}{\tau+s}$, (7) and (8) produce generalized conformable fractional integrals defined by Khan and Khan in 2019 [5].

(vi) If we take $g(x)=\frac{(x-a)^{s}}{s}, s>0$ in (7) and $g(x)=-\frac{(b-x)^{s}}{s}, s>0$ in (8), then conformable $(k, s)$-fractional integrals are achieved as defined by Habib et al. in 2018 [6].

(vii) If we take $g(x)=\frac{x^{1+s}}{1+s}$, then conformable fractional integrals are achieved as defined by Sarikaya et al. in 2016 [7].

(viii) If we take $g(x)=\frac{(x-a)^{s}}{s}, s>0$ in (7) and $g(x)=-\frac{(b-x)^{s}}{s}, s>0$ in (8) with $k=1$, then conformable fractional integrals are achieved as defined by Jarad et al. in 2017 [8].

In the upcoming section a generalized integral operator is constructed under suitable conditions on the utilized functions. Its two sided forms are investigated and it is shown that they are bounded. Furthermore, a new definition of integral operators has been presented. From the new definition it is shown that by proposing particular functions, Riemann-Liouville fractional integrals and all their generalizations can be achieved. Also bounds of all fractional and conformable integral operators presented in Remark 1 are deduced.

\section{Results and discussion}

Theorem 5. Let $f, \phi:[a, b] \longrightarrow \mathbb{R}, 0<a<b$, be positive and integrable functions. Let $g:[a, b] \longrightarrow \mathbb{R}$ be $a$ differentiable and increasing function. If $\frac{\phi}{x}$ is increasing on $[a, b]$, then for $x \in[a, b]$ we have

$$
\left|\int_{a}^{x} \frac{\phi(g(x)-g(t))}{g(x)-g(t)} g^{\prime}(t) f(t) d t\right| \leq \phi(g(b)-g(a))\|f\|_{[a, x]}
$$


and

$$
\left|\int_{x}^{b} \frac{\phi(g(t)-g(x))}{g(t)-g(x)} g^{\prime}(t) f(t) d t\right| \leq \phi(g(b)-g(a))|| f \|_{[x, b]} .
$$

Hence

$$
\left|\int_{a}^{x} \frac{\phi(g(x)-g(t))}{g(x)-g(t)} g^{\prime}(t) f(t) d t+\int_{x}^{b} \frac{\phi(g(t)-g(x))}{g(t)-g(x)} g^{\prime}(t) f(t) d t\right| \leq 2 \phi(g(b)-g(a))\|f\|_{[a, b]}
$$

Proof. As $g$ is increasing, therefore for $t \in[a, x) ; x \in[a, b], g(x)-g(t) \leq g(x)-g(a)$. The function $\frac{\phi}{x}$ is increasing, therefore one can obtain:

$$
\frac{\phi(g(x)-g(t))}{g(x)-g(t)} \leq \frac{\phi(g(x)-g(a))}{g(x)-g(a)} .
$$

It is given that $f$ is positive and $g$ is differentiable and increasing. Therefore from (10), the following inequality is valid:

$$
\frac{\phi(g(x)-g(t))}{g(x)-g(t)} g^{\prime}(t) f(t) \leq \frac{\phi(g(x)-g(a))}{g(x)-g(a)} g^{\prime}(t) f(t) .
$$

From which the following inequality can be obtained:

$$
\left|\int_{a}^{x} \frac{\phi(g(x)-g(t))}{g(x)-g(t)} g^{\prime}(t) f(t) d t\right| \leq \phi(g(b)-g(a))\|f\|_{[a, x]}
$$

Now on the other hand for $t \in(x, b]$ and $x \in[a, b]$, the following inequality holds true:

$$
\frac{\phi(g(t)-g(x))}{(g(t)-g(x))} g^{\prime}(t) f(t) \leq \frac{\phi(g(b)-g(x))}{(g(b)-g(x))} g^{\prime}(t) f(t)
$$

Hence (13), produces the following inequality:

$$
\left|\int_{x}^{b} \frac{\phi(g(t)-g(x))}{(g(t)-g(x))} g^{\prime}(t) f(t) d t\right| \leq \phi(g(b)-g(a))\|f\|_{[x, b]} .
$$

From (12) and (14), via triangular inequality (9) can be achieved.

Aforementioned theorem motivates to give the definition of a new two-sided integral operator as follows:

Definition 6. Let $f, g:[a, b] \longrightarrow \mathbb{R}, 0<a<b$, be the functions such that $f$ be positive and $f \in L_{1}[a, b]$, and $g$ be differentiable and increasing. Also let $\phi$ be a positive function such that $\frac{\phi}{x}$ is increasing on $[a, \infty)$. Then for $x \in[a, b]$ the left and right integral operators are defined by

$$
\left(F_{a^{+}}^{\phi, g} f\right)(x)=\int_{a}^{x} \frac{\phi(g(x)-g(t))}{g(x)-g(t)} g^{\prime}(t) f(t) d t
$$

and

$$
\left(F_{b^{-}}^{\phi, g} f\right)(x)=\int_{x}^{b} \frac{\phi(g(t)-g(x))}{g(t)-g(x)} g^{\prime}(t) f(t) d t
$$

Integral operators defined in (15) and (16) have connection with various fractional and conformable integral operators. This connection is shown in the following propositions and corollaries.

Proposition 7. Let $\phi(t)=\frac{t^{\alpha}}{\Gamma(\alpha)}, \alpha>0$. Then (15) and (16) produce the fractional integral operators (5) and (6), as follows:

$$
\left(F_{a^{+}}^{\frac{t^{\alpha}}{\Gamma(\alpha)}, g} f\right)(x):={ }_{g}^{\alpha} I_{a^{+}} f(x)
$$

and

$$
\left(F_{b^{-}}^{\frac{t^{\alpha}}{\Gamma(\alpha)}, g} f\right)(x):={ }_{g}^{\alpha} I_{b^{-}} f(x) .
$$


Further these operators satisfy the following bound for $\alpha \geq 1$ :

$$
\left|{ }_{g}^{\alpha} I_{a^{+}} f(x)+{ }_{g}^{\alpha} I_{b^{-}} f(x)\right| \leq \frac{2(g(b)-g(a))^{\alpha}}{\Gamma(\alpha)}\|f\|_{[a, b]} .
$$

Proposition 8. Let $g(x)=I(x)=x$. Then (15) and (16) produce integral operators defined by Sarikaya and Ertugral [8] as follows:

$$
\left(F_{a^{+}}^{\phi, I} f\right)(x):=\left({ }_{a^{+}} I_{\phi} f\right)(x)=\int_{a}^{x} \frac{\phi(x-t)}{(x-t)} f(t) d t
$$

and

$$
\left(F_{b^{-}}^{\phi, I} f\right)(x):=\left({ }_{b^{-}} I_{\phi} f\right)(x)=\int_{x}^{b} \frac{\phi(t-x)}{(t-x)} f(t) d t .
$$

Further these operators satisfy the following bound:

$$
\left|\left(a^{+} I_{\phi} f\right)(x)+\left({ }_{b}-I_{\phi} f\right)(x)\right| \leq 2 \phi(b-a)\|f\|_{[a, b]} .
$$

Moreover, the bounds of fractional and conformable integral operators associated with integral operators (15) and (16), are identified in the following corollaries:

Corollary 9. If we take $\phi(t)=\frac{\frac{\alpha}{k}}{k \Gamma_{k}(\alpha)}$. Then (15) and (16) produce the fractional integral operators (7) and (8) as follows:

$$
\left(F_{a^{+}}^{\frac{t^{\frac{\alpha}{k}}}{K_{\Gamma}(\alpha)}, g} f\right)(x):={ }_{g}^{\alpha} I_{a^{+}}^{k} f(x)
$$

and

$$
\left(F_{b^{-}}^{\frac{t^{\frac{\alpha}{k}}}{k \Gamma_{k}(\alpha)}, g} f\right)(x):={ }_{g}^{\alpha} I_{b^{-}}^{k} f(x) .
$$

From (9) the following bound holds for $\alpha \geq k$ :

$$
\left|{ }_{g}^{\alpha} I_{a^{+}}^{k} f(x)+{ }_{g}^{\alpha} I_{b^{-}}^{k} f(x)\right| \leq \frac{2(g(b)-g(a))^{\frac{\alpha}{k}}}{k \Gamma_{k}(\alpha)}\|f\|_{[a, b]} .
$$

Corollary 10. If we take $\phi(t)=\frac{t^{\alpha}}{\Gamma(\alpha)}, \alpha>0$ and $g(x)=I(x)=x$. Then (15) and (16) produce the fractional integral operators (1) and (2) as follows:

$$
\left(F_{a^{+}}^{\frac{t^{\alpha}}{\Gamma(\alpha)}, I} f\right)(x):={ }^{\alpha} I_{a^{+}} f(x)
$$

and

$$
\left(F_{b^{-}}^{\frac{t^{\alpha}}{\Gamma(\alpha)}, I} f\right)(x):={ }^{\alpha} I_{b^{-}} f(x) .
$$

From (9) the following bound holds for $\alpha \geq 1$ :

$$
\left|{ }^{\alpha} I_{a^{+}} f(x)+{ }^{\alpha} I_{b^{-}} f(x)\right| \leq \frac{2(b-a)^{\alpha}}{\Gamma(\alpha)}\|f\|_{[a, b]} .
$$

Corollary 11. If we take $\phi(t)=\frac{t^{\frac{\alpha}{k}}}{k \Gamma_{k}(\alpha)}$ and $g(x)=I(x)=x$. Then (15) and (16) produce the fractional integral operators (3) and (4) as follows:

and

$$
\left(F_{b^{-}}^{\frac{t^{\frac{\alpha}{k}}}{k \Gamma_{k}(\alpha)}, I} f\right)(x):={ }^{\alpha} I_{b^{-}}^{k} f(x) .
$$

From (9) the following bound holds for $\alpha \geq k$ :

$$
\left|{ }^{\alpha} I_{a^{+}}^{k} f(x)+{ }^{\alpha} I_{b^{-}}^{k} f(x)\right| \leq \frac{2(b-a)^{\frac{\alpha}{k}}}{k \Gamma_{k}(\alpha)}|f f|_{[a, b]} .
$$


Corollary 12. If we take $\phi(t)=\frac{t^{\alpha}}{\Gamma(\alpha)}, \alpha>0$ and $g(x)=\frac{x^{\rho}}{\rho}, \rho>0$. Then (15) and (16) produce the fractional integral operators defined by Chen and Katugampola [4] as follows:

$$
\left(F_{a^{+}}^{\frac{t^{\alpha}}{\Gamma(\alpha)}, g} f\right)(x)=\left({ }^{\rho} I_{a^{+}}^{\alpha} f\right)(x)=\frac{\rho^{1-\alpha}}{\Gamma(\alpha)} \int_{a}^{x}\left(x^{\rho}-t^{\rho}\right)^{\alpha-1} t^{\rho-1} f(t) d t
$$

and

$$
\left(F_{b^{-}}^{\frac{t^{\alpha}}{\Gamma(\alpha)}, g} f\right)(x)=\left({ }^{\rho} I_{b^{-}}^{\alpha} f\right)(x)=\frac{\rho^{1-\alpha}}{\Gamma(\alpha)} \int_{x}^{b}\left(t^{\rho}-x^{\rho}\right)^{\alpha-1} t^{\rho-1} f(t) d t .
$$

From (9), these operators satisfy the following bounds:

$$
\left|\left({ }^{\rho} I_{a^{+}}^{\alpha} f\right)(x)+\left({ }^{\rho} I_{b^{-}}^{\alpha} f\right)(x)\right| \leq \frac{2\left(b^{\rho}-a^{\rho}\right)^{\alpha}}{\rho^{\alpha} \Gamma(\alpha)}\|f\|_{[a, b]} .
$$

Corollary 13. If we take $\phi(t)=\frac{t^{\alpha}}{\Gamma(\alpha)}, \alpha>0$ and $g(x)=\frac{x^{s+1}}{s+1}, s>0$. Then (15) and (16) produce the fractional integral operators as follows:

$$
\left(F_{a^{+}}^{\frac{t^{\alpha}}{\Gamma(\alpha)}, g} f\right)(x)=\left({ }^{s} I_{a^{+}}^{\alpha} f\right)(x)=\frac{(s+1)^{1-\alpha}}{\Gamma(\alpha)} \int_{a}^{x}\left(x^{s+1}-t^{s+1}\right)^{\alpha-1} t^{s} f(t) d t
$$

and

$$
\left(F_{b^{-}}^{\frac{t^{\alpha}}{\Gamma(\alpha)}, g} f\right)(x)=\left({ }^{s} I_{b^{-}}^{\alpha} f\right)(x)=\frac{(s+1)^{1-\alpha}}{\Gamma(\alpha)} \int_{x}^{b}\left(t^{s+1}-x^{s+1}\right)^{\alpha-1} t^{s} f(t) d t .
$$

From (9), these operators satisfy the following bound:

$$
\left|\left({ }^{s} I_{a^{+}}^{\alpha} f\right)(x)+\left({ }^{s} I_{b^{-}}^{\alpha} f\right)(x)\right| \leq \frac{2\left(b^{s+1}-a^{s+1}\right)^{\alpha}}{(s+1)^{\alpha} \Gamma(\alpha)}\|f\|_{[a, b]} .
$$

Corollary 14. If we take $\phi(t)=\frac{t \frac{\alpha}{k}}{k \Gamma_{k}(\alpha)}, \alpha>k$ and $g(x)=\frac{x^{s+1}}{s+1}, s>0$. Then (15) and (16) produce the fractional integral operators defined by Sarikaya et al. [7] as follows:

$$
\left(F_{a^{+}}^{\frac{t^{\frac{\alpha}{k}}}{k \Gamma^{(\alpha)}}, g} f\right)(x)=\left({ }_{k}^{s} I_{a^{+}}^{\alpha} f\right)(x)=\frac{(s+1)^{1-\frac{\alpha}{k}}}{k \Gamma_{k}(\alpha)} \int_{a}^{x}\left(x^{s+1}-t^{s+1}\right)^{\frac{\alpha}{k}-1} t^{s} f(t) d t
$$

and

$$
\left(F_{b^{-}}^{\frac{t^{\frac{\alpha}{k}}}{k_{k}(\alpha)}, g} f\right)(x)=\left({ }_{k}^{s} I_{b^{-}}^{\alpha} f\right)(x)=\frac{(s+1)^{1-\frac{\alpha}{k}}}{k \Gamma_{k}(\alpha)} \int_{x}^{b}\left(t^{s+1}-x^{s+1}\right)^{\frac{\alpha}{k}-1} t^{s} f(t) d t .
$$

From (9), these operators satisfy the following bound:

$$
\left|\left({ }_{k}^{s} I_{a^{+}}^{\alpha} f\right)(x)+\left({ }_{k}^{s} I_{b^{-}}^{\alpha} f\right)(x)\right| \leq \frac{2\left(b^{s+1}-a^{s+1}\right)^{\frac{\alpha}{k}}}{(s+1)^{\frac{\alpha}{k} k} k \Gamma_{k}(\alpha)}|| f||_{[a, b]} .
$$

Corollary 15. If we take $\phi(t)=\frac{t^{\alpha}}{\Gamma(\alpha)}, \alpha>0$ and $g(x)=\frac{x^{\beta+s}}{\beta+s}, \beta, s>0$. Then (15) and (16) produce the fractional integral operators defined by Khan and Khan [5] as follows:

$$
\left(F_{a^{+}}^{\frac{t^{\alpha}}{\Gamma(\alpha)}, g} f\right)(x)=\left({ }_{\beta}^{s} I_{a^{+}}^{\alpha} f\right)(x)=\frac{(\beta+s)^{1-\alpha}}{\Gamma(\alpha)} \int_{a}^{x}\left(x^{\beta+s}-t^{\beta+s}\right)^{\alpha-1} t^{s} f(t) d_{\beta} t
$$

and

$$
\left(F_{b^{-}}^{{\frac{t^{\alpha}}{\alpha}}^{\alpha}, g} f\right)(x)=\left({ }_{\beta}^{s} I_{b^{-}}^{\alpha} f\right)(x)=\frac{(\beta+s)^{1-\alpha}}{\Gamma(\alpha)} \int_{x}^{b}\left(t^{\beta+s}-x^{\beta+s}\right)^{\alpha-1} t^{s} f(t) d_{\beta} t
$$


From (9), these operators satisfy the following bound:

$$
\left|\left({ }_{\beta}^{s} I_{a^{+}}^{\alpha} f\right)(x)+\left({ }_{\beta}^{s} I_{b^{-}}^{\alpha} f\right)(x)\right| \leq \frac{2\left(b^{\beta+s}-a^{\beta+s}\right)^{\alpha}}{(\beta+s)^{\alpha} \Gamma(\alpha)}\|f\|_{[a, b]} .
$$

Corollary 16. If we take $g(x)=\frac{(x-a)^{\rho}}{\rho}, \rho>0$ in (15) and $g(x)=\frac{-(b-x)^{\rho}}{\rho}, \rho>0$ in (16) with $\phi(t)=\frac{t^{\alpha}}{\Gamma(\alpha)}, \alpha>0$. Then (15) and (16) produce the fractional integral operators defined by Jarad et al. [9] as follows:

$$
\left(F_{a^{+}}^{\frac{t^{\alpha}}{\Gamma(\alpha)}, g} f\right)(x)=\left({ }^{\rho} I_{a^{+}}^{\alpha} f\right)(x)=\frac{\rho^{1-\alpha}}{\Gamma(\alpha)} \int_{a}^{x}\left((x-a)^{\rho}-(t-a)^{\rho}\right)^{\alpha-1}(t-a)^{\rho-1} f(t) d t
$$

and

$$
\left(F_{b^{-}}^{\frac{t^{\alpha}}{\Gamma(\alpha)}, g} f\right)(x)=\left({ }^{\rho} I_{b^{-}}^{\alpha} f\right)(x)=\frac{\rho^{1-\alpha}}{\Gamma(\alpha)} \int_{x}^{b}\left((b-x)^{\rho}-(b-t)^{\rho}\right)^{\alpha-1}(b-t)^{\rho-1} f(t) d t .
$$

From (9), these operators satisfy the following bound:

$$
\left|\left({ }^{\rho} I_{a^{+}}^{\alpha} f\right)(x)+\left({ }^{\rho} I_{b^{-}}^{\alpha} f\right)(x)\right| \leq \frac{2(b-a)^{\rho \alpha}}{\rho^{\alpha} \Gamma(\alpha)}\|f\|_{[a, b]} .
$$

Corollary 17. If we take $g(x)=\frac{(x-a)^{\rho}}{\rho}, \rho>0$ in (15) and $g(x)=\frac{-(b-x)^{\rho}}{\rho}, \rho>0$ in (16) with $\phi(t)=\frac{t^{\frac{\alpha}{k}}}{k \Gamma_{k}(\alpha)}, \alpha>k$. Then (15) and (16) produce the fractional integral operators defined by Habib et al. [6] as follows:

$$
\left(F_{a^{+}}^{\frac{t^{\frac{\alpha}{k}}}{k(\alpha)}, g} f\right)(x)=\left({ }_{k}^{\rho} I_{a^{+}}^{\alpha} f\right)(x)=\frac{\rho^{1-\frac{\alpha}{k}}}{k \Gamma_{k}(\alpha)} \int_{a}^{x}\left((x-a)^{\rho}-(t-a)^{\rho}\right)^{\frac{\alpha}{k}-1}(t-a)^{\rho-1} f(t) d t
$$

and

$$
\left(F_{b^{-}}^{\frac{t^{\frac{\alpha}{k}}}{k \Gamma_{k}(\alpha)}, g} f\right)(x)=\left({ }_{k}^{\rho} I_{b^{-}}^{\alpha} f\right)(x)=\frac{\rho^{1-\frac{\alpha}{k}}}{k \Gamma_{k}(\alpha)} \int_{x}^{b}\left((b-x)^{\rho}-(b-t)^{\rho}\right)^{\frac{\alpha}{k}-1}(b-t)^{\rho-1} f(t) d t .
$$

From (9), these operators satisfy the following bound:

$$
\left|\left({ }_{k}^{\rho} I_{a^{+}}^{\alpha} f\right)(x)+\left({ }_{k}^{\rho} I_{b^{-}}^{\alpha} f\right)(x)\right| \leq \frac{2(b-a)^{\frac{\rho \alpha}{k}}}{\rho^{\frac{\alpha}{k}} k \Gamma_{k}(\alpha)}|| f \|_{[a, b]} .
$$

\section{Conclusion}

In this paper the author formulate a generalized integral operator as well as its boundedness. The investigated integral operator produces almost all fractional and conformable integral operators which have been discovered by the authors of recent decades. Furthermore, bounds of all these fractional integrals can be obtained. The existence of new integral operators may be useful in the further investigations of different aspects of functional analysis and operator theory etc.

Conflicts of Interest: "The author declare no conflict of interest."

\section{References}

[1] Mubeen, S., \& Habibullah, G. M. (2012). $k$-fractional integrals and applications. International Journal of Contemporary Mathematical Sciences, 7, 89-94.

[2] Kilbas, A. A., Srivastava, H. M., \& Trujillo, J. J. (2006). Theory and applications of fractional differential equations. First edition, pp. 99. North-Holland Mathematics Studies, 204, Elsevier, New York-London.

[3] Kwun, Y. C., Farid, G., Nazeer, W., Ullah, S., \& Kang, S. M. (2018). Generalized Riemann-Liouville $k$-fractional integrals associated with Ostrowski type inequalities and error bounds of Hadamard Inequalities. IEEE access, 6 , 64946-64953.

[4] Chen, H., \& Katugampola, U. N. (2017). Hermite-Hadamard and Hermite-Hadamard-Fejér type inequalities for generalized fractional integrals. Journal of Mathematical Analysis and Applications, 446, 1274-1291.

[5] Khan, T. U., \& Khan M. A. (2019). Generalized conformable fractional operators. Journal of Computational and Applied Mathematics, 346, 378-389. 
[6] Habib, S., Mubeen, S., \& Naeem, M. N. (2018). Chebyshev type integral inequalities for generalized $k$-fractional conformable integrals. Journal of Inequalities and Special Functions, 9(4), 53-65.

[7] Sarikaya, M. Z., Dahmani, M., Kiris, M. E., \& Ahmad, F. (2016). (k,s)-Riemann-Liouville fractional integral and applications. Hacettepe Journal of Mathematics and Statistics, 45(1), 77-89.

[8] Sarikaya, M. Z., \& Ertuğral, F. (2017). On the generalized Hermite-Hadamard inequalities. https:/ / www.researchgate.net/publication/321760443.

[9] Jarad, F., Ugurlu, E., Abdeljawad, T., \& Baleanu, D. (2017). On a new class of fractional operators. Advances in Difference Equations, 2017(1), 247.

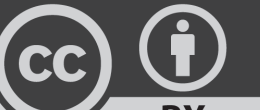

BY

(C) 2019 by the authors; licensee PSRP, Lahore, Pakistan. This article is an open access article distributed under the terms and conditions of the Creative Commons Attribution (CC-BY) license (http://creativecommons.org/licenses/by/4.0/). 